1 Hacettepe Journal of Mathematics and Statistics

$\bigcap$ Volume 46 (4) (2017), 603-611

\title{
The first Zagreb and forgotten topological indices of $d$-ary trees
}

\author{
Ramin Kazemi*† and Ali Behtoei ${ }^{\dagger}$
}

\begin{abstract}
The first Zagreb index of a graph is equal to the sum of the square of the vertex degrees of the graph and the forgotten topological index (Findex) of a graph is defined as the sum of cubes of the vertex degrees of the graph. These parameters have applications in chemistry and drug structures. For any fixed integer $d \geq 2$, the $d$-ary tree is a rooted tree in which each node has no more than $d$ children. We determine the mean value of these indices in $d$-ary trees. Also, we obtain some useful relations and equalities related to these indices and other graphical parameters.
\end{abstract}

Keywords: First Zagreb index, Forgotten topological index, $d$-ary tree, Mean. 2000 AMS Classification: 60C05, 05C05, 05C07.

Received : 05.10.2016 Accepted : 13.11.2016 Doi : 10.15672/HJMS.20174622758

\section{Introduction}

Let $G$ be a connected graph. Two vertices of $G$ which are connected by an edge are said to be adjacent. The number of vertices adjacent to a given vertex $v$ is the degree of $v$ and is denoted by $d_{v}$ (or $\left.d(v)\right)$. A chemical graph is a graph whose vertices denote atoms and edges denote bonds between those atoms of the underlying chemical structure. A topological index for a (chemical) graph $G$ is a numerical quantity invariant under automorphisms of $G$ and it does not depend on the labeling or pictorial representation of the graph. Topological indices and graph invariants based on the distances between vertices of a graph or vertex degrees are widely used for characterizing molecular graphs, establishing relationships between structure and properties of molecules, predicting biological activity of chemical compounds, and making their chemical applications. When

\footnotetext{
*Department of Statistics, Imam Khomeini International University, P. O. Box: 34149-16818, Qazvin, Iran, Email: r.kazemi@sci.ikiu.ac.ir

†Corresponding Author.

†Department of Mathematics, Imam Khomeini International University, P. O. Box: 3414916818, Qazvin, Iran, Email: a.behtoei@sci.ikiu.ac.ir
} 
analyzing structure-dependency of total $\pi$-electron energy [10], an approximate formula was obtained in which terms of the form

$$
Z_{2}(G)=\sum_{v \in V(G)} d_{v}^{2}
$$

where $V(G)$ is the vertex set of a graph $G$. It was immediately recognized that this term increase with the increasing extent of branching of the carbon-atom skeleton, i.e., that this provide quantitative measure of molecular branching. Ten years later, $Z_{2}$ was included among topological indices and was named as Zagreb group index [1]. The name Zagreb group index was soon abbreviated to Zagreb index, and nowadays $Z_{2}$ is referred to as the first Zagreb index. Horoldagva and Das in [11] compared two Zagreb indices and studied special kinds of graphs for which some conjectured relations on Zagreb indices hold for them. Yang et al. studied Zagreb coindices of some composite graphs [14]. The research background of the Zagreb index together with its generalization appears in chemistry or mathematical chemistry (see for examples: [1], [3] and [9] and references therein).

Followed by the first Zagreb index, Furtula and Gutman [7] introduced forgotten topological index (also called F-index) which was defined as

$$
Z_{3}(G)=\sum_{v \in V(G)} d_{v}^{3}
$$

Furtula and Gutman [7] raised that the predictive ability of forgotten topological index is almost similar to that of first Zagreb index and for the acentric factor and entropy, and both of them obtain correlation coefficients larger than 0.95 . This fact implies the reason why forgotten topological index is useful for testing the chemical and pharmacological properties of drug molecular structures. Sun et al. [13] deduced some basic nature of forgotten topological index and reported that this index can reinforce the physicochemical flexibility of Zagreb indices. Recently, Gao et al. [8] manifested the forgotten topological index of some significant drug molecular structures.

Che and Chen [2] provided new lower and upper bounds of the forgotten topological index in terms of graph irregularity, Zagreb indices, graph size, and maximum/minimum vertex degrees. They characterized all graphs that attain the new bounds of F-index and showed that the new bounds are better than the bounds given in [7] for all benzenoid systems with more than one hexagon. As corollaries, various upper bounds of F-index easily follow. Moreover, upper bounds for connected $K_{r+1}$-free graphs are also presented.

Let $F_{v}$ be a function dependent of a vertex of vertices of the molecular graph $G$. Došlić et al. [4] established a general identity for topological indices of the type $T(G)=$ $\sum_{v \in V(G)} F_{v}$, which we state as follows:

1.1. Theorem. Let $G$ be any connected graph, and any of its invariants satisfying $T(G)=\sum_{v \in V(G)} F_{v}$. Then

$$
T(G)=\sum_{u v \in E(G)}\left(\frac{F_{u}}{d_{u}}+\frac{F_{v}}{d_{v}}\right),
$$

where $E(G)$ is the edge set of $G$.

Note that using Theorem 1.1 we have

$$
Z_{2}(G)=\sum_{u v \in E(G)}\left(d_{u}+d_{v}\right)
$$

and

$$
Z_{3}(G)=\sum_{u v \in E(G)}\left(d_{u}^{2}+d_{v}^{2}\right) .
$$


Figure 1. A tree $T$ with its node degrees indicated. In tree $T, Z_{2}(T)=$ 54 and $Z_{3}(T)=160$.

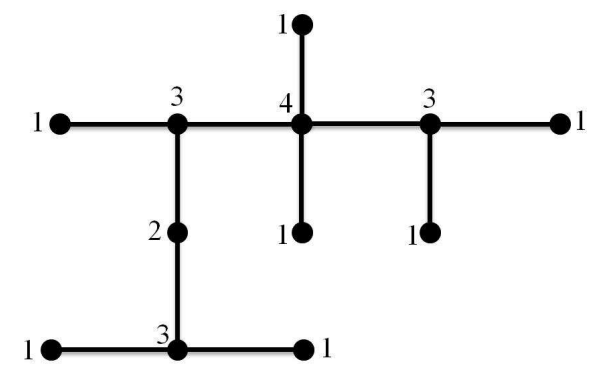

The paper is organized as follows. In Section 2 we give the evolution processes of the ordinary and $d$-ary trees. In Section 3 , we compute the exact values $\mathbb{E}\left(Z_{2, n}\right)$ and $\mathbb{E}\left(Z_{3, n}\right)$ (the mean of the first Zagreb index and forgotten index, respectively) for ordinary trees and $d$-ary trees of order $n$.

\section{Evolution process}

The structures of many molecules such as dendrimers, alkanes and acyclic molecules are tree like. Rooted trees have wide applications in chemical graph theory such as enumeration and coding problems of chemical structures. Structures of chemical compounds can be synthesized and categorized through mathematical means. Chemists have a long tradition of using atomic valences (vertex degrees) to find molecular structures graphically. Almost all of rooted chemical trees are special kinds of $d$-ary trees for $d=4$. Connections of chemistry to random trees have been investigated by many researchers. We present the following evolution processes for random trees of order $n$, which turns out to be appropriate when studying the Zagreb indices of trees.

Ordinary trees: Every tree of order $n$ can be obtained uniquely by attaching $n$th node to one of the $n-1$ nodes in a tree of order $n-1$. It is of particular interest in applications to assume the random tree model and to speak about a random tree with $n$ nodes, which means that all trees of order $n$ are considered to appear equally likely. Equivalently one may describe random trees via the following tree evolution process, which generates random trees of arbitrary order $n$. At step 1 the process starts with the root. At step $i$ the $i$ th node is attached to any previous node $v$ of the already grown tree $T$ of order $i-1$ with probability $p_{i}(v)=\frac{1}{i-1}$. For applicability of our own results and specially connection with the chemical relevance, see [12]. An illustrative example is provided in Figure 1.

$d$-ary trees: For any fixed integer $d \geq 2$, the $d$-ary tree is a rooted tree in which each node has no more than $d$ children. The possible insertion possitions to join a new node to a $d$-ary tree are called external nodes. In a $d$-ary tree, the number of nodes can be attached to node $v$ of out-degree $\bar{d}_{v}$ is $d-\bar{d}_{v}$. Therefore the number of all external nodes in a $d$-ary tree $T$ of order $n$ is

$$
\sum_{v \in V(T)}\left(d-\bar{d}_{v}\right)=(d-1) n+1
$$


At step 1 the process starts with the root. At step $i$ the $i$ th node is attached to a previous node $v$ of the already grown $d$-ary tree $T$ of order $i-1$ with probability

$$
p_{i}(v)=\frac{d-\bar{d}_{v}}{(d-1)(i-1)+1} .
$$

It is obvious that $\bar{d}_{\text {root }}=d_{\text {root }}$ and for other vertices $\bar{d}_{v}=d_{v}-1$. Thus

$$
p_{i}(\text { root })=\frac{d-d_{\text {root }}}{(d-1)(i-1)+1}
$$

and for other vertices

$$
p_{i}(v)=\frac{d-d_{v}+1}{(d-1)(i-1)+1} .
$$

Let $Z_{2, n}$ be the first Zagreb index of a random tree of order $n$ and $\mathcal{F}_{n}$ be the sigma-field generated by the first $n$ stages of these trees [12]. Let $U_{n}$ be a randomly chosen node belonging to a tree of order $n$. Also, let $Z_{3, n}$ be the forgotten topological index of a random tree of order $n$.

\section{The main results}

\subsection{Ordinary trees.}

3.1. Theorem. For a rooted tree of order $n$,

$$
\mathbb{E}\left(Z_{2, n}\right)=6(n-1)-4 H_{n-1},
$$

where $H_{n}$ is the $n$-th harmonic number.

Proof. By definition,

$$
Z_{2, n}=Z_{2, n-1}+\left(d_{U_{n-1}}+1\right)^{2}-d_{U_{n-1}}^{2}+1=Z_{2, n-1}+2 d_{U_{n-1}}+2 .
$$

Hence,

$$
\begin{aligned}
\mathbb{E}\left(Z_{2, n} \mid \mathcal{F}_{n-1}\right) & =\mathbb{E}\left(Z_{2, n-1}+2 d_{U_{n-1}}+2 \mid \mathcal{F}_{n-1}\right) \\
& =Z_{2, n-1}+2 \mathbb{E}\left(d_{U_{n-1}} \mid \mathcal{F}_{n-1}\right)+2 \\
& =Z_{2, n-1}+2 \frac{1}{n-1} \sum_{i=1}^{n-1} d_{v_{i}}+2 \\
& =Z_{2, n-1}+2 \frac{2(n-2)}{n-1}+2 \\
& =Z_{2, n-1}+6-\frac{4}{n-1},
\end{aligned}
$$

since $Z_{2, n-1}$ is $\mathcal{F}_{n-1}$-measurable. We have

$$
\begin{aligned}
\mathbb{E}\left(Z_{2, n}\right) & =\mathbb{E}\left(Z_{2, n-1}\right)+6-\frac{4}{n-1} \\
& =\left(\mathbb{E}\left(Z_{2, n-2}\right)+6-\frac{4}{n-2}\right)+6-\frac{4}{n-1} \\
& \vdots \\
& =\mathbb{E}\left(Z_{2,1}\right)+(n-1) 6-4\left(\frac{1}{n-1}+\frac{1}{n-2}+\cdots+1\right) \\
& =(n-1) 6-4 H_{n-1},
\end{aligned}
$$

since $Z_{2,1}=0$.

We note that Theorem 3.1 also holds for the random recursive trees, see [5]. 
3.2. Theorem. For each rooted tree of order $n$, we have

$$
\mathbb{E}\left(Z_{3, n}\right)=26(n-1)-24 H_{n-1}-6 H_{n-1}^{2}+6 H_{n-1}^{(2)},
$$

where $H_{n}^{(2)}$ is the $n$-th harmonic number of order 2.

Proof. Using the definition of forgotten topological index and by the stochastic growth rule of the tree, we see that

$$
Z_{3, n}=Z_{3, n-1}+\left(d_{U_{n-1}}+1\right)^{3}-d_{U_{n-1}}^{3}+1=Z_{3, n-1}+3 d_{U_{n-1}}^{2}+3 d_{U_{n-1}}+2 .
$$

This implies that

$$
\begin{aligned}
\mathbb{E}\left(Z_{3, n} \mid \mathcal{F}_{n-1}\right) & =Z_{3, n-1}+3 \mathbb{E}\left(d_{U_{n-1}}^{2} \mid \mathcal{F}_{n-1}\right)+3 \mathbb{E}\left(d_{U_{n-1}} \mid \mathcal{F}_{n-1}\right)+2 \\
& =Z_{3, n-1}+\frac{3}{n-1} \sum_{k=1}^{n-1} d_{v_{k}}^{2}+\frac{3}{n-1} \sum_{k=1}^{n-1} d_{v_{k}}+2 \\
& =Z_{3, n-1}+\frac{3}{n-1} Z_{2, n-1}+\frac{3}{n-1}(2(n-2))+2 \\
& =Z_{3, n-1}+\frac{3}{n-1} Z_{2, n-1}+8-\frac{6}{n-1}
\end{aligned}
$$

Thus,

$$
\mathbb{E}\left(Z_{3, n}\right)=\mathbb{E}\left(Z_{3, n-1}\right)+\frac{3}{n-1} \mathbb{E}\left(Z_{2, n-1}\right)+8-\frac{6}{n-1} .
$$

Therefore, Theorem 3.1 implies that

$$
\begin{aligned}
\mathbb{E}\left(Z_{3, n}\right) & =\mathbb{E}\left(Z_{3, n-1}\right)+\frac{3}{n-1}\left(6(n-2)-4 H_{n-2}\right)+8-\frac{6}{n-1} \\
& =\mathbb{E}\left(Z_{3, n-1}\right)+26-\frac{24}{n-1}-\frac{12}{n-1} H_{n-2} \\
& \vdots \\
& =\mathbb{E}\left(Z_{3,1}\right)+(n-1) 26-24 H_{n-1}-12 \sum_{k=1}^{n-1} \frac{H_{k}}{k}+12 \sum_{k=1}^{n-1} \frac{1}{k^{2}} .
\end{aligned}
$$

This completes the proof because $Z_{3,1}=0$ and $\sum_{k=1}^{n} \frac{H_{k}}{k}=\frac{1}{2}\left(H_{n}^{2}+H_{n}^{(2)}\right)$.

\section{2. $d$-ary trees.}

Let

$$
\bar{Z}_{2, n}=\sum_{v \in V(T)} \bar{d}_{v}^{2}, \quad \bar{Z}_{3, n}=\sum_{v \in V(T)} \bar{d}_{v}^{3} .
$$

Now using the stochastic growth rule of the tree, we see that

$$
\bar{Z}_{2, n}=\bar{Z}_{2, n-1}+\left(\bar{d}_{U_{n-1}}+1\right)^{2}-\bar{d}_{U_{n-1}}^{2}=\bar{Z}_{2, n-1}+2 \bar{d}_{U_{n-1}}+1
$$

3.3. Lemma. For each d-ary tree of order $n$, we have

$$
Z_{2, n}=\bar{Z}_{2, n}-2 \bar{d}_{\text {root }}+3(n-1)
$$

and

$$
Z_{3, n}=\bar{Z}_{3, n}+3\left(\bar{Z}_{2, n}-\bar{d}_{\text {root }}^{2}-\bar{d}_{\text {root }}\right)+4(n-1) .
$$


Proof. Using definition we obtain

$$
\begin{aligned}
Z_{2, n} & =\sum_{v \in V(T)} d_{v}^{2} \\
& =\bar{d}_{\text {root }}^{2}+\sum_{v \neq \text { root }}\left(\bar{d}_{v}+1\right)^{2} \\
& =\bar{d}_{\text {root }}^{2}+\sum_{v \neq \text { root }}\left(\bar{d}_{v}^{2}+2 \bar{d}_{v}+1\right) \\
& =\bar{Z}_{2, n}+2\left(n-1-\bar{d}_{\text {root }}\right)+(n-1) \\
& =\bar{Z}_{2, n}-2 \bar{d}_{\text {root }}+3(n-1) .
\end{aligned}
$$

The second statement can be similarly obtained.

For each $n, d \geq 2$ let $q_{n}=n(d-1)+1$ and using the gamma function define

$$
\beta_{n, i}=\frac{\Gamma\left(\frac{n d-n+1}{d-1}\right)}{\Gamma\left(\frac{n d-n-i+1}{d-1}\right)}, \quad i \geq 1 .
$$

3.4. Lemma. For each $k \geq 1$, we have $1-\frac{k}{q_{n-1}}=\frac{\beta_{n-1, k}}{\beta_{n, k}}$.

Proof. Since $\Gamma(x)=(x-1) \Gamma(x-1)$, the proof is obvious and straightforward [6].

3.5. Theorem. Let $\bar{d}_{U_{n}}$ be the out-degree of node $U_{n}$ in a d-ary tree of order $n$. Then

$$
\mathbb{E}\left(\bar{d}_{U_{n}}\right)=\frac{1}{\beta_{n, 1}} \sum_{i=1}^{n-1} \beta_{i+1,1} \lambda_{i, d}
$$

and

$$
\mathbb{E}\left(\vec{d}_{U_{n}}^{2}\right)=\frac{1}{\beta_{n, 2}} \sum_{i=1}^{n-1} \beta_{i+1,2} \eta_{i, d}
$$

where $\lambda_{n, d}=\frac{d}{q_{n}}$ and

$$
\eta_{n, d}=\frac{2 d-1}{q_{n}} \mathbb{E}\left(\bar{d}_{U_{n}}\right)+\lambda_{n, d} .
$$

Proof. It is obvious that

$$
\mathbb{E}\left(\bar{d}_{U_{n}}=\bar{d}_{U_{n-1}}+1 \mid \mathcal{F}_{n-1}\right)=\frac{d-\bar{d}_{U_{n-1}}}{q_{n-1}}
$$

and

$$
\mathbb{E}\left(\bar{d}_{U_{n}}=\bar{d}_{U_{n-1}} \mid \mathcal{F}_{n-1}\right)=1-\frac{d-\bar{d}_{U_{n-1}}}{q_{n-1}} .
$$

Thus

$$
\begin{aligned}
\mathbb{E}\left(\bar{d}_{U_{n}} \mid \mathcal{F}_{n-1}\right) & =\left(\bar{d}_{U_{n-1}}+1\right) \times \frac{d-\bar{d}_{U_{n-1}}}{q_{n-1}}+\bar{d}_{U_{n-1}} \times\left(1-\frac{d-\bar{d}_{U_{n-1}}}{q_{n-1}}\right) \\
& =\left(1-\frac{1}{q_{n-1}}\right) \bar{d}_{U_{n-1}}+\lambda_{n-1, d} \\
& =\frac{\beta_{n-1,1}}{\beta_{n, 1}} \bar{d}_{U_{n-1}}+\lambda_{n-1, d} .
\end{aligned}
$$


Hence

$$
\begin{aligned}
\mathbb{E}\left(\bar{d}_{U_{n}}\right) & =\frac{\beta_{n-1,1}}{\beta_{n, 1}} \mathbb{E}\left(\bar{d}_{U_{n-1}}\right)+\lambda_{n-1, d} \\
& \vdots \\
& =\frac{1}{\beta_{n, 1}} \sum_{i=1}^{n-1} \beta_{i+1,1} \lambda_{i, d}
\end{aligned}
$$

since $\mathbb{E}\left(\bar{d}_{U_{1}}\right)=0$. Also,

$$
\begin{aligned}
\mathbb{E}\left(\bar{d}_{U_{n}}^{2} \mid \mathcal{F}_{n-1}\right) & =\left(\bar{d}_{U_{n-1}}+1\right)^{2} \times \frac{d-\bar{d}_{U_{n-1}}}{q_{n-1}}+\bar{d}_{U_{n-1}}^{2} \times\left(1-\frac{d-\bar{d}_{U_{n-1}}}{q_{n-1}}\right) \\
& =\left(1-\frac{2}{q_{n-1}}\right) \bar{d}_{U_{n-1}}^{2}+(2 d-1) \frac{\bar{d}_{U_{n-1}}}{q_{n-1}}+\lambda_{n-1, d}
\end{aligned}
$$

Hence

$$
\begin{aligned}
\mathbb{E}\left(\bar{d}_{U_{n}}^{2}\right) & =\left(1-\frac{2}{q_{n-1}}\right) \mathbb{E}\left(\bar{d}_{U_{n-1}}^{2}\right)+(2 d-1) \frac{\mathbb{E}\left(\bar{d}_{U_{n-1}}\right)}{q_{n-1}}+\lambda_{n-1, d} \\
& \vdots \\
& =\frac{1}{\beta_{n, 2}} \sum_{i=1}^{n-1} \beta_{i+1,2} \eta_{i, d}
\end{aligned}
$$

For each $n, d \geq 2$ define

$$
\alpha_{n, d}=\frac{2 d(n-1)}{q_{n}}+1, \sigma_{n, d}=\frac{3(d-1)}{q_{n}} \mathbb{E}\left(\bar{Z}_{2, n}\right)+\frac{3}{2} \alpha_{n, d}+1 .
$$

3.6. Theorem. For each d-ary tree of order $n$, we have

$$
\mathbb{E}\left(Z_{2, n}\right)=\frac{1}{\beta_{n, 2}} \sum_{i=1}^{n-1} \beta_{i+1,2} \alpha_{i, d}-\frac{2}{\beta_{n, 1}} \sum_{i=1}^{n-1} \beta_{i+1,1} \lambda_{i, d}+3(n-1) .
$$

Proof. The equality $\bar{Z}_{2, n}=\bar{Z}_{2, n-1}+2 \bar{d}_{U_{n-1}}+1$ implies that

$$
\begin{aligned}
\mathbb{E}\left(\bar{Z}_{2, n} \mid \mathcal{F}_{n-1}\right) & =\bar{Z}_{2, n-1}+\frac{2}{q_{n-1}} \sum_{k=1}^{n-1} \bar{d}_{v_{k}}\left(d-\bar{d}_{v_{k}}\right)+1 \\
& =\left(1-\frac{2}{q_{n-1}}\right) \bar{Z}_{2, n-1}+\alpha_{n-1, d} \\
& =\frac{\beta_{n-1,2}}{\beta_{n, 2}} \bar{Z}_{2, n-1}+\alpha_{n-1, d}
\end{aligned}
$$

Thus

$$
\mathbb{E}\left(\bar{Z}_{2, n}\right)=\frac{\beta_{n-1,2}}{\beta_{n, 2}} \mathbb{E}\left(\bar{Z}_{2, n-1}\right)+\alpha_{n-1, d} .
$$

Proof is completed by Lemma 3.3. Note that $Z_{2,1}=0$.

3.7. Theorem. For each d-ary tree of order $n$,

$$
\mathbb{E}\left(Z_{3, n}\right)=\sum_{i=1}^{n-1}\left(\frac{\beta_{i+1,3} \sigma_{i, d}}{\beta_{n, 3}}+\frac{3 \beta_{i+1,2}\left(\alpha_{i, d}-\eta_{i, d}\right)}{\beta_{n, 2}}-\frac{3 \beta_{i+1,1} \lambda_{i, d}}{\beta_{n, 1}}\right)+4(n-1) .
$$


Proof. Using the equality $\bar{Z}_{3, n}=\bar{Z}_{3, n-1}+3 \bar{d}_{U_{n-1}}^{2}+3 \bar{d}_{U_{n-1}}+1$ we have

$$
\begin{aligned}
\mathbb{E}\left(\bar{Z}_{3, n} \mid \mathcal{F}_{n-1}\right) & =\bar{Z}_{3, n-1}+3 \mathbb{E}\left(\bar{d}_{U_{n-1}}^{2} \mid \mathcal{F}_{n-1}\right)+3 \mathbb{E}\left(\bar{d}_{U_{n-1}} \mid \mathcal{F}_{n-1}\right)+1 \\
& =\bar{Z}_{3, n-1}+3 \sum_{i=1}^{n-1} \frac{d-\bar{d}_{v_{i}}}{q_{n-1}} \bar{d}_{v_{i}}^{2}+3 \sum_{i=1}^{n-1} \frac{d-\bar{d}_{v_{i}}}{q_{n-1}} \bar{d}_{v_{i}}+1 \\
& =\bar{Z}_{3, n-1}-\frac{3}{q_{n-1}} \bar{Z}_{3, n-1}+\frac{3 d}{q_{n-1}} \bar{Z}_{2, n-1}-\frac{3}{q_{n-1}} \bar{Z}_{2, n-1} \\
& +\frac{3 d(n-2)}{q_{n-1}}+1 \\
& =\left(1-\frac{3}{q_{n-1}}\right) \bar{Z}_{3, n-1}+\frac{3(d-1)}{q_{n-1}} \bar{Z}_{2, n-1}+\frac{3 d(n-2)}{q_{n-1}}+1 .
\end{aligned}
$$

Then

$$
\mathbb{E}\left(\bar{Z}_{3, n}\right)=\frac{\beta_{n-1,3}}{\beta_{n, 3}} \mathbb{E}\left(\bar{Z}_{3, n-1}\right)+\sigma_{n-1, d} .
$$

By Lemma 3.3 proof is completed.

3.8. Theorem. Suppose

$$
Z_{k, n}=\sum_{v \in V(T)} d_{v}^{k}, \quad k \in \mathbb{N} .
$$

Then for a rooted tree $T$ of order $n$,

$$
(n-1)\left((1+\delta)^{k-1}+1\right) \leq \mathbb{E}\left(Z_{k, n}\right) \leq(n-1)\left((1+\Delta)^{k}+1\right),
$$

where $\delta$ and $\Delta$ are the minimum degree and the maximum degree of $T$, respectively.

Proof. By definition,

$$
Z_{k, n}=Z_{k, n-1}+\sum_{j=0}^{k-1}\left(\begin{array}{l}
k \\
j
\end{array}\right) d_{U_{n-1}}^{j}+1
$$

Hence,

$$
\mathbb{E}\left(Z_{k, n} \mid \mathcal{F}_{n-1}\right)=Z_{k, n-1}+\frac{1}{n-1} \sum_{j=0}^{k-1} \sum_{i=1}^{n-1}\left(\begin{array}{l}
k \\
j
\end{array}\right) d_{v_{i}}^{j}+1,
$$

since $Z_{k, n-1}$ is $\mathcal{F}_{n-1}$-measurable. Proof is completed since $\delta \leq d_{v_{i}} \leq \Delta$ and $Z_{k, 1}=0$.

\section{Acknowledgements}

We thank the reviewers for their helpful suggestions, which improved our manuscript.

\section{References}

[1] Balaban, A.T. and Motoc, I. and Bondchev, D. and Mekenyan, O. Topological indices for structure-activity correlations, Topics Curr. Chem. 114, 21-55, 1983.

[2] Che, Z. and Chen, Z. Lower and upper bounds of the forgotten topological index, MATCH Commun. Math. Comput. Chem. 76 (3), 635-648, 2016.

[3] Das, K.C. and Gutman, I. Bounds for the energy of graphs, Hacet. J. Math. Stat. 45 (3), 695-703, 2016.

[4] Došlić, T. and Réti, T. and Vukičević, D. On the vertex degree indices of connected graphs, Chem. Phys. Lett. 512, 283-286, 2011.

[5] Feng, Q. and Hu, Z. On the Zagreb index of random recursive trees, J. Appl. Prob. 48 1189-1196, 2011.

[6] Feng, Q. and Hu, Z. Asymptotic normality of the Zagreb index of random b-ary recursive trees, Far Eastern Math. J. 15(1) 91-101, 2015. 
[7] Furtula, B. and Gutman, I. A forgotten topological index, J. Math. Chem. 53 (4), 1184-1190, 2015.

[8] Gao, W. and Farahani, M.R. and Shi, L. Forgotten topological index of some drug structures, Acta Med. Medit., in press, 2016.

[9] Gutman, I. and Furtula, B. Estimating the second and third geometric-arithmetic indices, Hacet. J. Math. Stat. 40 (1), 69-76 , 2011.

[10] Gutman, I. and Trinajstić, N. Graph theory and molecular orbitals. Total $\pi$-electron energy of alternant hydrocarbons, Chem. Phys. Lett. 17, 535-538, 1972.

[11] Horoldagva, B. and Das, K.C. On comparing Zagreb indices of graphs, Hacet. J. Math. Stat. 41 (2), 223-230, 2012.

[12] Kazemi, R. The second Zagreb index of molecular graphs with tree structure, MATCH Commun. Math. Comput. Chem. 72, 753-760, 2014.

[13] Sun, Y.C. and Lin, Z. and Peng, W.X. and Yuan, T.Q. and Xu. F. and Wu, Y.Q. and Yang, J. and Wang, Y.S. and Sun, R.C. Chemical changes of raw materials and manufactured binderless boards during hot pressing: lignin isolation and characterization, Bioresources $\mathbf{9}$ (1), 1055-1071, 2014.

[14] Yang, L. and Ai, X. and Zhang, L. The Zagreb coindices of a type of composite graph, Hacet. J. Math. Stat. 45 (4), 1135-1142, 2016. 
